\title{
Calculation of Short Circuit Based on Compensation Method
}

\author{
Feng GAO* \\ Beijing Kedong Electric Control System Co. Ltd \\ Simulation application department \\ Beijing, China \\ E-mail: yl_10000@sina.com \\ +* Corresponding author \\ Huijing BI \\ State Grid Hebei Power Company \\ Training Centre \\ Shijiazhuang, China \\ E-mail: 14280004@qq.com
}

\author{
Xilan ZHAO \\ State Grid Gansu Electric Power Company \\ Dispatch department \\ Tianshui, China \\ E-mail: kailuojuanshu@163.com
}

Gang HUANG

State Grid Liaoning Power Company

Training Centre

Dalian, China

E-mail: zzj5818@qq.com

\begin{abstract}
In order to improve the shortcomings of short circuit current of traditional power grid, this paper presents a method based on compensation method for short circuit current calculation. In the algorithm, the compensating method is used to introduce the compensation current in the fault port, so as to simulate the influence of the node short circuit fault on the original network. The method uses the admittance matrix before the fault to solve the state of the network, without modifying the node admittance matrix, can solve the whole network. This method can save the time of the admittance matrix after solving the fault, and can calculate the short circuit faults of various types. The calculation speed is fast and the result is accurate and reliable, which can meet the requirements of large grid adjustment and optimization of short circuit current. The method can provide technical support for the analysis of short circuit current limiting strategy.
\end{abstract}

Keywords-transient stability; symmetric component method; compensation method; short circuit calculation

\section{INTRODUCTION}

An important question in the power system transient stability analysis [1,2,3] is how to handle faults and operations, especially asymmetric faults, single-phase reclosing, and other asymmetric operations, As well as the same time in the power grid complex fault processing.

Traditional fault handling methods have the following problems:

Since the node needs to be added at the point of failure, it is necessary to correct the node number of the original faulty line endpoint. After the fault disappears, clear the node and can't use the node admittance matrix that has been formed before the failure occurs. Considering that there are different trips and coincidence on both sides of the lateral fault in the line, more than one node is added to this faulty line. It is too cumbersome to deal with, and the calculation efficiency is not high.
In this paper, the use of compensation method to deal with fault problems, to avoid the above problems, the process is simple, can handle very complex multiple failures.

\section{PRINCIPLE OF COMPENSATION}

The use of compensation method [4,5] for fault calculation, to avoid the traditional fault algorithm complex problems, the concept of clear and intuitive, fast calculation, can handle complex failures. The schematic diagram is as follows:

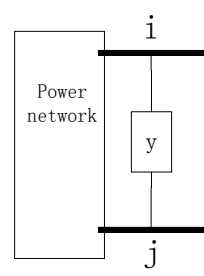

(a)

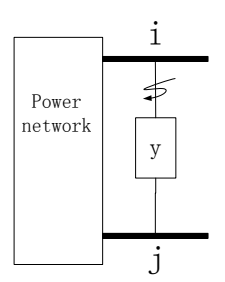

(b)

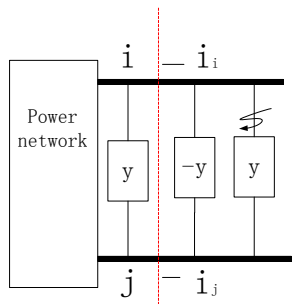

(c)
Figure 1. Schematic diagram of fault calculation principle

In Fig. 1, (a) shows the power grid before the fault, and a fault occurs between nodes $\mathrm{i}$ and $\mathrm{j}$. (B) indicates that $\mathrm{a}$ transverse or longitudinal fault has occurred between nodes $i$ and $\mathrm{j}$, and the admittance of line ij is $\mathrm{y}$. (B) and (c) show that the two lines of admittance between $y$ and $-\mathrm{y}$ are connected in parallel between nodes $\mathrm{i}$ and $\mathrm{j}$, and their equivalent admittance is zero, Is equivalent. In (c) the red dotted line on the left side of the network is restored before the failure, the impact of the current $\mathrm{i} \neg \mathrm{i}$ and $\mathrm{ij}$ said, the equivalent of the original network nodes $\mathrm{i}$ and $\mathrm{j}$ were injected into the current ii and ij.

The power network in Fig. 1 represents the positive sequence, the negative sequence, and the zero sequence network, respectively. Although the fault point can't use the symmetric component method to decouple the three order power, but the failure of the three order networks can be used node $\mathrm{i}$, $\mathrm{j}$ three order injection current representation. 
From Fig. 1 (c), the red dotted lines are left and right respectively. To the left, the current-voltage relationship can be composed of fault port impedance matrix, in fact, the node impedance matrix in this port element. To the right, the relationship between current and voltage can form a boundary condition matrix. Two sets of equations can solve the fault current and voltage.

As the red dotted line on the left side of the positive and negative zero sequence network is decoupled, so easy to write positive and negative zero sequence six current voltage equation. Although the right side can't decouple positive and negative zero sequence current and voltage relationship, but can write abc three series current and voltage matrix relationship, and then converted to positive and negative zero sequence power. This is a total of twelve equations, node $\mathrm{i}$ and node $\mathrm{j}$ positive and negative zero sequence current voltage a total of twelve unknowns, so the equation can be solved.

In the actual solution process, to solve the red dotted line right boundary condition matrix, because the matrix dimension is very small, so the calculation speed is very fast. However, when solving the node impedance matrix related elements on the left side of the red dotted line, it is timeconsuming to calculate the inverse of the inverse matrix from the node admittance matrix.

\section{FAULT PORT IMPEDANCE ARRAY}

The faulty impedance array is the matrix information of the nodes that are contracted to the fault-related nodes before the fault occurs. In fact, the matrix of the node impedance matrix is composed of the relevant elements of the faulty port.

Before the occurrence of the fault, there is already positive and negative zero sequence network, with the impedance matrix in the form of the following:

Positive sequence:

$$
\underline{Z}^{(1)} \dot{I}^{(1)}=-\dot{U}_{0}^{(1)}+\stackrel{\dot{U}^{(1)}}{ }
$$

Negative sequence:

$$
\underline{Z}^{(2)} \dot{\bullet}^{(2)}=\dot{U}^{(2)}
$$

Zero sequence:

$$
\underline{Z}^{(0)} \stackrel{\bullet}{I^{(0)}}=\dot{U^{(0)}}
$$

$\underline{Z}^{(1)}, \underline{Z}^{(2)}, \underline{Z}^{(0)}$ Respectively, means $\mathrm{n} \times \mathrm{n}$ order ( $\mathrm{n}$ is the number of network nodes) positive sequence, negative sequence and zero sequence node impedance matrix, $\dot{I^{(1)}}, \dot{I^{(2)}}, \dot{I^{(0)}}$ Respectively, that n-dimensional positive sequence, negative sequence and zero sequence node injection current vector, $\dot{U^{(1)}}, \dot{U^{(2)}}, \dot{U^{(0)}}$ Respectively, that n-dimensional positive sequence, negative sequence and zero sequence node voltage vector, $\dot{U}_{0}^{(1)}$ Represents the n-dimensional node positive sequence initial voltage.

The current vectors $\dot{I^{(1)}}, \dot{\dot{(2)}^{(2)}}, \dot{(0)}^{(0)}$ are injected at the above nodes ,Only the number of nodes associated with the fault is not zero, the other is zero. So the current vector multiplied by the node impedance matrix, and only retain the fault-related nodes of the line, you can get the shrink matrix, the fault port impedance array.

The fault-related node voltage is:

$$
\dot{U}_{F}=\left[\begin{array}{llllll}
u_{i}^{(0)} & \dot{u_{i}^{(1)}} & \dot{u_{i}^{(2)}} & \dot{u_{j}^{(0)}} & \dot{u_{j}^{(1)}} & \dot{u}_{j}^{(2)}
\end{array}\right]^{T} \text {, }
$$

The fault-related node injection current is:

$$
\dot{I_{F}}=\left[\begin{array}{cccccc}
\dot{i_{i j}^{(0)}} & \dot{i_{i j}^{(1)}} & \dot{i}_{i j}^{(2)} & \dot{i_{j i}^{(0)}} & \dot{i}_{j i}^{(1)} & \dot{i}_{j i}^{(2)}
\end{array}\right]^{T},
$$

The initial voltage of the fault-related node is:

$$
\dot{U_{F 0}}=\left[\begin{array}{llllll}
0 & \dot{u_{i 0}^{(1)}} & 0 & 0 & \dot{u_{j 0}^{(1)}} & 0
\end{array}\right]^{T} \text {, }
$$

There are:

$$
\underline{Z}_{D} \dot{I}_{F}=-\dot{U}_{F 0}+\dot{U}_{F}
$$

There $\underline{Z}_{D}$ is the faulty port impedance array, $\underline{Z}_{D}$ is:

$$
\underline{Z}_{D}=\left[\begin{array}{cccccc}
z_{i i}^{(0)} & 0 & 0 & z_{i j}^{(0)} & 0 & 0 \\
0 & z_{i i}^{(1)} & 0 & 0 & z_{i j}^{(1)} & 0 \\
0 & 0 & z_{i i}^{(2)} & 0 & 0 & z_{i j}^{(2)} \\
z_{j i}^{(0)} & 0 & 0 & z_{j j}^{(0)} & 0 & 0 \\
0 & z_{j i}^{(1)} & 0 & 0 & z_{j j}^{(1)} & 0 \\
0 & 0 & z_{j i}^{(2)} & 0 & 0 & z_{j j}^{(2)}
\end{array}\right] .
$$

\section{BOUNDARY CONDITION MATRIX SOLUTION}

From Fig. 1 (c) in the red dotted line to the right to see two branches, the right is the compensation branch; the 
middle is the compensation branch, respectively, can establish the corresponding matrix.

(1) Fault branch boundary condition matrix

For the various faults in the line, as long as the fault point abc write the current and voltage relations, can be simply converted to positive and negative zero sequence current and voltage relationship.

As shown in Fig. 2, the k-point single-phase ground fault occurs at point $\mathrm{k}$, assuming that the ground admittance is $\mathrm{yk}$.

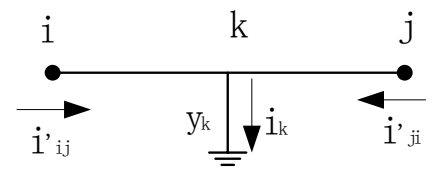

Figure 2. Boundary condition matrix formation specification

Then $\mathrm{k}$ point abc power relationship is as follows:

$$
\left[\begin{array}{c}
i_{k a} \\
i_{k b} \\
i_{k c}
\end{array}\right]=\underline{Y_{k}}\left[\begin{array}{l}
u_{k a} \\
u_{k b} \\
u_{k c}
\end{array}\right]
$$

Among them

$$
\underline{Y_{k}}=\left[\begin{array}{ccc}
y_{k} & 0 & 0 \\
0 & 0 & 0 \\
0 & 0 & 0
\end{array}\right] .
$$

Positive and negative zero sequence and abc power relationship is as follows:

$$
\left[\begin{array}{l}
u_{a} \\
u_{b} \\
u_{c}
\end{array}\right]=\underline{A}\left[\begin{array}{l}
u_{0} \\
u_{1} \\
u_{2}
\end{array}\right]
$$

$$
\text { Among them } \begin{aligned}
\underline{A} & =\left[\begin{array}{ccc}
1 & 1 & 1 \\
1 & a^{2} & a \\
1 & a & a^{2}
\end{array}\right], \\
a & =e^{j 120^{\circ}}=-\frac{1}{2}+j \frac{\sqrt{3}}{2},
\end{aligned}
$$

$$
a^{2}=e^{j 240^{\circ}}=-\frac{1}{2}-j \frac{\sqrt{3}}{2}
$$

$$
\underline{A}^{-1}=\frac{1}{3}\left[\begin{array}{ccc}
1 & 1 & 1 \\
1 & a & a^{2} \\
1 & a^{2} & a
\end{array}\right] .
$$

Then abc relationship becomes positive and negative zero sequence relationship, as follows:

$$
\left[\begin{array}{l}
i_{k a} \\
i_{k b} \\
i_{k c}
\end{array}\right]=\underline{Y}\left[\begin{array}{l}
u_{k a} \\
u_{k b} \\
u_{k c}
\end{array}\right] \rightarrow \underline{A}\left[\begin{array}{c}
i_{k 0} \\
i_{k 1} \\
i_{k 2}
\end{array}\right]=\underline{Y} \underline{A}\left[\begin{array}{l}
u_{k 0} \\
u_{k 1} \\
u_{k 2}
\end{array}\right] \rightarrow\left[\begin{array}{c}
i_{k 0} \\
i_{k 1} \\
i_{k 2}
\end{array}\right]=\underline{A^{-1}} \underline{Y A}\left[\begin{array}{l}
u_{k 0} \\
u_{k 1} \\
u_{k 2}
\end{array}\right] \rightarrow\left[\begin{array}{c}
i_{k 0} \\
i_{k 1} \\
i_{k 2}
\end{array}\right]=\underline{Y}\left[\begin{array}{l}
u_{k 0} \\
u_{k 1} \\
u_{k 2}
\end{array}\right]
$$

\section{Among them}

$$
\underline{Y}_{k}=\underline{A}^{-1} \underline{Y} \underline{A}=\frac{1}{3}\left[\begin{array}{ccc}
1 & 1 & 1 \\
1 & a & a^{2} \\
1 & a^{2} & a
\end{array}\right]\left[\begin{array}{ccc}
y_{k} & 0 & 0 \\
0 & 0 & 0 \\
0 & 0 & 0
\end{array}\right]\left[\begin{array}{ccc}
1 & 1 & 1 \\
1 & a^{2} & a \\
1 & a & a^{2}
\end{array}\right]=\frac{y_{k}}{3}\left[\begin{array}{ccc}
1 & 1 & 1 \\
1 & 1 & 1 \\
1 & 1 & 1
\end{array}\right]
$$

Since the positive and negative zero sequences are decoupled in the ik and $\mathrm{jk}$ segments in Fig. 2, the relationship between the voltage and current of the node i node $\mathrm{j}$ is easily written by the relationship between $\mathrm{k}$ and $\mathrm{i}$ and $\mathrm{j}$.

$$
\dot{I}_{F}^{\prime}=\underline{Y}_{\Omega}^{\prime} \dot{U}_{F}
$$

Among them,

$$
\stackrel{I_{F}^{\prime}}{\prime}=\left[\begin{array}{cccccc}
\dot{i_{i j}^{(0)}} & \dot{i_{i j}^{(1)}} & \dot{i_{i j}^{(2)}} & \dot{i_{j i}^{(0)}} & \dot{i_{j i}^{(1)}} & \dot{i_{j i}^{(2)}}
\end{array}\right]^{T}
$$

is the fault current branch of the first branch of the current matrix, $\dot{U}_{F}$ is the fault-related node voltage matrix, $\underline{Y}_{\Omega}$ is the fault branch boundary condition matrix, its value is:

$$
\underline{Y}_{\Omega}^{\prime}=\left[\begin{array}{cc}
\underline{E}_{3 \times 3}+\underline{Y}_{k} \underline{Z}_{i k} & \underline{E}_{3 \times 3} \\
\underline{E}_{3 \times 3} & \underline{E}_{3 \times 3}+\underline{Y}_{k} \underline{Z}_{j k}
\end{array}\right]^{-1}\left[\begin{array}{cc}
\underline{Y}_{k} & \underline{0} \\
\underline{0} & \underline{Y}_{k}
\end{array}\right],
$$


Among them

$$
\begin{gathered}
\underline{E}_{3 \times 3}=\left[\begin{array}{ccc}
1 & 0 & 0 \\
0 & 1 & 0 \\
0 & 0 & 1
\end{array}\right], \underline{Z}_{i k}=\left[\begin{array}{ccc}
Z_{i k}^{(0)} & 0 & 0 \\
0 & z_{i k}^{(1)} & 0 \\
0 & 0 & z_{i k}^{(2)}
\end{array}\right], \\
\underline{Z}_{j k}=\left[\begin{array}{ccc}
z_{j k}^{(0)} & 0 & 0 \\
0 & z_{j k}^{(1)} & 0 \\
0 & 0 & z_{j k}^{(2)}
\end{array}\right] .
\end{gathered}
$$

(2)Compensation tributary boundary condition matrix

The middle branch is compensated branch, the branch impedance is -zij, you can write the compensation branch of the voltage and current relationship:

$$
{\stackrel{\bullet}{I_{F}}}_{F}=\underline{Y}_{\Omega}^{\prime \prime} \dot{U}_{F}
$$

In the above equation, $I_{F}^{\prime \prime}$ is the compensation branch sequence current, $\dot{U}_{F}$ is the fault node voltage, $\underline{Y}^{\prime \prime} \Omega$ is the compensation branch boundary matrix:

$$
\underline{Y}_{\Omega}^{\prime \prime}=\left[\begin{array}{cccccc}
\frac{1}{-z_{i j}^{(0)}} & 0 & 0 & -\frac{1}{-z_{i j}^{(0)}} & 0 & 0 \\
0 & \frac{1}{-z_{i j}^{(1)}} & 0 & 0 & -\frac{1}{-z_{i j}^{(1)}} & 0 \\
0 & 0 & \frac{1}{-z_{i j}^{(2)}} & 0 & 0 & -\frac{1}{-z_{i j}^{(2)}} \\
-\frac{1}{-z_{i j}^{(0)}} & 0 & 0 & \frac{1}{-z_{i j}^{(0)}} & 0 & 0 \\
0 & -\frac{1}{-z_{i j}^{(1)}} & 0 & 0 & \frac{1}{-z_{i j}^{(1)}} & 0 \\
0 & 0 & -\frac{1}{-z_{i j}^{(2)}} & 0 & 0 & \frac{1}{-z_{i j}^{(2)}}
\end{array}\right],
$$

$$
Z_{i j}^{(k)}(k=0,1,2) \text { is the sequence impedance of the fault }
$$

branch in Fig. 1 (c).

(3)Boundary condition matrix

The formulas (7) and (8) are combined to:

$$
\dot{I}_{F}^{\prime}+\dot{I}_{F}^{\prime}=\left(\underline{Y}_{\Omega}^{\prime}+\underline{Y}_{\Omega}^{\prime \prime}\right) \dot{U}_{F}
$$

$$
\text { Let } \dot{I}_{F}=-\left(\dot{I}_{F}^{\prime}+\dot{I}_{F}\right) \text {, then let } \underline{Y}_{\Omega}=-\left(\underline{Y}_{\Omega}^{\prime}+\underline{Y}_{\Omega}^{\prime \prime}\right) \text {, }
$$
then the formula (9) is:

$$
\dot{I}_{F}=\underline{Y}_{\Omega} \dot{U}_{F}
$$

(4) and (10), can solve the node i and node j positive and negative zero sequence voltage and current.

\section{Positive Order Equivalent Admittance ARRAY}

The concept of classical positive order equivalent admittance (or impedance) is to find the correlation coefficient of positive sequence voltage and positive sequence current at the point of failure, ie, positive order equivalent admittance (or impedance). Here we can expand this concept, is to find the fault-related nodes of the positive sequence injection current vector and positive sequence voltage vector correlation matrix, it may be called positive order equivalent admittance matrix. (4) and (10), eliminating the zero sequence and negative sequence, get the positive sequence voltage and current relationship matrix.

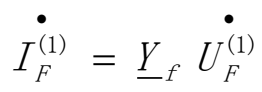

There $\mathrm{A}$ is the positive sequence current, $\mathrm{B}$ is the positive sequence voltage, $C$ is the joint (4) and (7), eliminating the zero sequence and the negative order of the equivalent admittance array.

And then the fault-induced positive order equivalent admittance matrix is superimposed on the electromechanical transient calculation of the node admittance matrix, can be included in the failure of electromechanical transient calculation of the impact.

In the realization of the program, the generated positive sequence equivalent admittance matrix is decomposed into several branches and admittance, and then into the node admittance matrix as the ordinary branch.

Set the admittance matrix of the network before the fault occurs, and the corrected admittance matrix is:

$$
\underline{Y}^{\prime}=\underline{Y}-\underline{Y}_{f}
$$

With the modified network admittance matrix, you can carry out electromechanical transient calculation, the calculation process and failure before the same.

\section{SUMMARY}

The traditional fault calculation method is to add a new node in the horizontal fault, the port is from the new node and the ground to see; in the vertical fault on each side of the addition of a new node, the port is from the two new nodes to see. The solution process is also from the port to look outward, that is, to see the fault, get the relationship between voltage and current; look inward, that is, to the fault-free grid to see, positive and negative zero sequence network decoupling current and voltage relationship. According to the type of fault, it is divided into string and type fault. And then the fault-tolerant positive and negative zero-order network decoupling the current and voltage relationship, in accordance with the string type or type of fault and the sum 
of the three orders of the voltage or the sum of the threeorder current is zero, forming the equation and solution.

In contrast, the compensation method of fault calculation has the following advantages:

Instead of adding a new node, it is possible to solve the three-order network impedance matrix element at one time, use it in multiple calculations (for instantaneous failures and coincidence success). Do not add new nodes, back to the programming to bring convenience, the concept of clear, simple operation.

Regardless of the horizontal fault or the longitudinal fault, only the boundary condition matrix consisting of the associated node voltage on both sides of the fault and the node injection current is required.

Do not use the ideal transformer to phase shift the power, the impact of different effects reflected in the boundary conditions array.

You can calculate the actual occurrence of any complex faults, including a line on both the occurrence of horizontal failure and different trips and other coincidence; you can consider the zero sequence in the group of cross-line fault.

\section{REFERENCES}

[1] Zhao Xiaoming.Research on Substation Modeling and Application of Protection Based of Electromagnetic Simulation System. Beijing: North China Electric Power University, 2015.

[2] Wu Wenhui, Cao Xianglin. Power System Electromagnetic Transient Calculation and EMTP Application. Beijing: China Water Resources and Hydropower Press, 2012.

[3] Zhai Pei, Yu Qingguang, Li Chengxin et al. Real-Time Electromechanical Transient Simulation of Power System. Power System Technology, 2008,32 (9): 42-45.

[4] Sun Y, Hang N S, Sun Z Y. Power flow calculation method forislanded power network. Power and Energy Engineering Conference, APPEEC 2009. Wuhan: IEEE, 2009: 1-5.

[5] Dan YangQing, Liu Wenying, Zhu Yanwei, et al. A Fast algorithm of short-circuit based on compensation method. Power System Automation, 2011,35 (14): 103-106. 